\title{
Análisis de los potenciales efectos en el reconocimiento de los activos de las pequeñas empresas de Cúcuta- Colombia por la implementación de la NIIF para Pymes ${ }^{1}$
}

\section{Analysis of the Potential effects on the recognition of the assets of small businesses in Cucuta - Colombia by the implementation of the IFRS for SMEs}

\section{Análise dos efeitos potenciais sobre o reconhecimento dos activos de empresas de pequeno porte em Cúcuta Colômbia para a implementação do IFRS para PMEs}

\author{
Eduardo Solano-Becerra ${ }^{1}$
}

Forma de citar: E. Solano-Becerra, "Análisis de los potenciales efectos en el reconocimiento de los activos de las pequeñas empresas de Cúcuta- Colombia por la implementación de la NIIF para Pymes”, Respuestas, vol. 22, no. 2, pp. 116-130, 2017.

Recibido:

Febrero 20 de 2017

Aceptado:

Mayo 18 de 2017

\section{Resumen}

Antecedentes: Ley 1314 de 2009 en la cual Colombia converge a estándares internacionales de aceptación mundial. Objetivo: El presente artículo realiza una descripción cualitativa de los potenciales efectos que podría generar la implementación de la NIIF para PYMES en relación con los requisitos para el reconocimiento de los activos. Métodos: El trabajo se desarrolló por medio de una metodología descriptiva, orientada a los cambios de la nueva normatividad contable aceptada en Colombia. Se tomó una muestra de 9 pequeñas empresas que mediante una metodología basada en los conceptos y principios de la NIIF para Pymes y se identificaron los elementos del activo que cumplían con la definición y reconocimiento de la misma. Resultados: Los resultados permitieron afirmar que la NIIF para Pymes hace hincapié en la importancia del análisis financiero de los bienes conforme a la realidad económica. Conclusión: Los efectos en cuanto a reconocimiento de los activos de las pequeñas empresas se reflejaron principalmente en el grupo de propiedad planta y equipo, diferidos, intangibles y valorizaciones.

Palabras Clave: Activos, Contabilidad, NIIF, Pymes, Reconocimiento.
${ }^{1}$ Magíster en Administración de Empresas eduardosolano@ufps.edu.co Orcid: 0000-0002-6702-1047

Docente de Planta del

Departamento de Ciencias Contables y Financieras de la Universidad Francisco de Paula Santander Cúcuta, Colombia

\begin{abstract}
Background: Law 13142009 in which Colombia converges to international standards for worldwide acceptance. Objective: This article is a qualitative description of the potential effects that could lead to the implementation of the IFRS for SMEs in relation to the requirements for the recognition of assets. Methods: The work was developed through a descriptive methodology, oriented to changes in new accounting standards accepted in Colombia. Took a sample of 9 small businesses that identified the elements of the asset that met the definition and recognition of the same using a methodology based on the concepts and principles of the IFRS for SMEs. Results: The results allowed to affirm that the IFRS for SMEs emphasizes the importance of the financial analysis of the goods in accordance with the economic reality. Conclusion: The effects in terms of recognition
\end{abstract}


of the assets of small businesses is mainly reflected in the group of property, plant and equipment, deferred, intangible and revaluation.

Keywords: Accounting, IFRS and SMEs, Assets, Recognition.
Julio - Dic. 2017

ISSN 0122-820X

E-ISSN 2422-5053

PP: $116-130$

\section{Resumo}

Antecedentes: Lei 1.314 de 2009 na qual a Colômbia convergir para os padrões internacionais aceites em todo o mundo. Objetivo: O presente artigo faz uma descrição qualitativa dos efeitos potenciais que poderiam levar à implementação de IFRS para PMEs em relação aos requisitos para reconhecimento de ativos. Métodos: O estudo foi realizado por um mudanças orientadas metodologia descritiva das novas normas de contabilidade aceitos na Colômbia. uma amostra de 9 pequenas empresas foi observado que o uso de uma metodologia baseada nos conceitos e princípios do IFRS para as PME e os ativos que se enquadram na definição e reconhecimento de que foram identificados. Resultados: Os resultados permitiram afirmar que o IFRS para PMEs enfatiza a importância da análise financeira da propriedade de acordo com a realidade econômica. Conclusão: Os efeitos em termos de reconhecimento dos ativos das pequenas empresas são refletidas principalmente no grupo de bens do ativo imobilizado, diferido, intangíveis e apreciações.

Palavras-chave: Ativo, contabilidade, IFRS, As PME, Reconhecimento.

\section{Introducción}

La contabilidad colombiana ha estado orientada por el marco normativo reglamentado por el decreto 2649 [1] y 2650 [2] de 1993, que en su momento fue tomado de los estándares internacionales de contabilidad e información financiera y se adaptó a las necesidades propias del entorno nacional.

Colombia no ha sido ajena a los estándares internacionales (NIC y NIIF) en diciembre 30 de 1999 la ley 550 [3] resalta en su artículo 63 la necesidad de que las normas contables colombianas se armonizaran con los usos y reglas internacionales, así mismo el proyecto de ley de intervención económica [4] presentada en diciembre 30 de 2003, pretendió la adopción en Colombia de los estándares internacionales de contabilidad, auditoría, contaduría y buen gobierno y finalmente el proyecto de ley 165 de 2007 [5] por el cual el estado colombiano adopta las normas internacionales de información financiera para la presentación de informes contables. Lo expuesto deja en evidencia las iniciativas para la adopción de estas normas, sin embargo la falta de respuesta a la problemática no permitieron que entraran en vigencia.

Dado este panorama, dicha normatividad aplicable no se fue actualizando, y como resultado el país quedo atrás de las tendencias internacionales. Por otro lado, el marco normativo no fue puesto en práctica en su totalidad, las normas tributarias y de control, en busca de información clara y verificable fueron modificando los principios contables.

Finalmente, estos hechos sirvieron como antecedente para aprobar la ley 1314 de 2009 [6] en la cual Colombia converge a estándares internacionales de aceptación mundial. Seguidamente el Consejo Técnico de la Contaduría Pública - CTCP como organismo rector de la normatividad contable, emite el Direccionamiento Estratégico de diciembre 
Análisis de los potenciales efectos en el reconocimiento de los activos de las pequeñas empresas de CúcutaColombia por la implementación de la NIIF para Pymes

5 de 2012 [7] identificando los estándares internacionales aplicables en Colombia y los tres grupos de empresas: grupo 1 que aplica NIIF Plenas, Grupo 2 que aplica NIIF para Pymes y Grupo 3 que aplica NIF para Microempresas.

En el caso de las Pymes el Ministerio de Comercio, Industria y Turismo- MCIT expidió el decreto 3022 de 2013 [8] en el cual reglamenta el marco técnico normativo para los preparadores de información financiera que conforman el grupo 2. Considerando por recomendación del CTCP tomar como referente la Norma Internacional de Información Financiera para Pequeñas y
Medianas Entidades-NIIF para Pymes (en inglés International Financial Reporting Standard-IFRS for SMEs) conformada por treinta y cinco (35) secciones, y que fueron expedidas en el 2009 por el Consejo de Normas Internacionales de Contabilidad (en inglés International Accounting Estándares Board-IASB). Así mismo, fija el cronograma de aplicación del marco técnico normativo de información financiera y las características que deben cumplir las entidades para ser clasificadas dentro de este grupo.

En la tabla I se muestra un resumen de las generalidades normativas aplicables al grupo 2 que aplica NIIF para Pymes.

Tabla I. Generalidades del nuevo marco técnico normativo para el grupo 2 que aplican NIIF para PYMES

\begin{tabular}{|c|c|c|c|}
\hline CRONOGRAMA & \multicolumn{2}{|c|}{ APLICAN NIIF PARA PYMES } & $\begin{array}{l}\text { LIMITES LEY } \\
590 \text { DE } 2004\end{array}$ \\
\hline $\begin{array}{l}\text { Fecha de expedición del Marco Técnico Normativo: } 27 \text { de } \\
\text { diciembre de } 2013\end{array}$ & \multirow[b]{2}{*}{ Empresas pequeñas o medianas } & Activos & $>500$ SMLMV \\
\hline $\begin{array}{c}\text { Periodo de preparación obligatoria: periodo para que las } \\
\text { pymes preparen el proceso de convergencia a las nuevas } \\
\text { normas. Enero } 1 \text { al } 31 \text { de diciembre de } 2014\end{array}$ & & Ingresos & $>6.000 \mathrm{SMLMV}$ \\
\hline $\begin{array}{l}\text { Presentación Estado de Situación Financiera de Apertura- } \\
\text { ESFA: este estado financiero medirá por primera vez los } \\
\text { activos, pasivos y el patrimonio de la entidad, bajo el Marco } \\
\text { Técnico Normativo para las pymes. Enero } 1^{\circ} \text { de } 2015\end{array}$ & \multirow{3}{*}{$\begin{array}{c}\text { - Empresas Grandes que no sea matriz } \\
\text { ni subordinada de } \\
\text { empresa nacional o extranjera } \\
\text { que aplique NIIF Plenas } \\
\text { - No realicen importaciones o } \\
\text { exportaciones que representen más del } \\
50 \% \text { de las compras o de las ventas } \\
\text { respectivamente }\end{array}$} & Activos & $\begin{array}{l}>30.000 \\
\text { SMLMV }\end{array}$ \\
\hline $\begin{array}{c}\text { Periodo de transición: las pymes deberán llevar la } \\
\text { contabilidad bajo decreto } 2649 \text { y } 2650 \text { de } 1993 \text {, en paralelo } \\
\text { a la construcción de la información contable aplicando el } \\
\text { nuevo marco técnico normativo, decreto } 3022 \text { de } 2013 \text {. } \\
\text { Enero } 1 \text { al } 31 \text { de diciembre de } 2015\end{array}$ & & \multirow[b]{2}{*}{ Planta de Personal } & \multirow[b]{2}{*}{$\begin{array}{l}>200 \\
\text { Trabajadores }\end{array}$} \\
\hline $\begin{array}{c}\text { Fecha de presentación últimos estados financieros: } \\
\text { presentación últimos estados financieros elaborados } \\
\text { conforme a los decretos } 2649 \text { y } 2650 \text { de } 1993.31 \text { de } \\
\text { diciembre de } 2015\end{array}$ & & & \\
\hline $\begin{array}{c}\text { Periodo de aplicación: se llevará por primera vez la } \\
\text { contabilidad bajo el Nuevo Marco Técnico Normativo. } \\
\text { Enero } 1 \text { al } 31 \text { de diciembre de } 2016\end{array}$ & Microempresa & Ingresos & $>6.000 \mathrm{SMMLV}$ \\
\hline $\begin{array}{l}\text { Fecha de reporte: primeros estados financieros } \\
\text { comparativos: Diciembre } 31 \text { de } 2016\end{array}$ & \multicolumn{3}{|c|}{$\begin{array}{l}\text { Al Grupo } 2 \text { pertenecerán quienes no cumplan con los requisitos para pertenecer al Grupo } 1 \text { o } \\
\text { al Grupo } 3\end{array}$} \\
\hline
\end{tabular}

Fuente: elaboración propia a partir de actualícese.com [9]

Posteriormente la superintendencia de sociedades mediante la circular externa 115000002 de 2014 [10] emitió instrucciones que facilitaran la aplicación del decreto 3022 de 2013, que en el 2014 fue modificado por el decreto 2267 de 2014 [11] en los artículos 1 y 2 del mismo. Finalmente el decreto 2420 de 2015 [12] compila los decretos reglamentarios de la Ley 1314 del 2009 con respecto a NIIF y NAI: decretos 2706 del 2012, 2784 del 2012, 3022 del 2013 y 302 del 2015. La figura 1 muestra la ruta normativa para la implementación de la NIIF para Pymes en Colombia. 


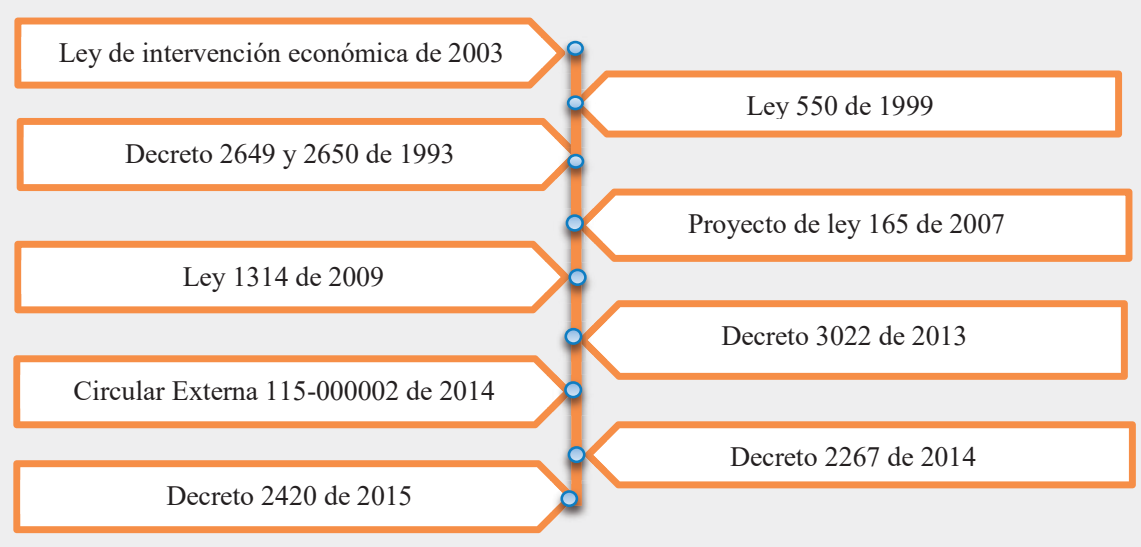

Figura 1. Reglamentación de implementación de la NIIF para PYMES grupo 2.

En este orden de ideas, y frente a la necesidad de conocer el efecto que estas normas pueden tener en los activos de las pequeñas empresas. El presente artículo describe los resultados de un estudio cualitativo, sobrela implementación de la NIIF para Pymes, específicamente en el reconocimiento de los activos de una muestra de pequeñas empresas de Cúcuta, que cumplen con los requisitos mencionados en la tabla I. En el primer capítulo se describe la literatura apreciable que ha estudiado los efectos por la implementación de la NIIF para Pymes, identificando principalmente el comportamiento de los activos, que permitirá guiar los resultados de la investigación y ser contrastados con la literatura referenciada e identificar si los efectos son similares o no, dado que las características por tamaño, NIIF a aplicar y sector son diferentes. En el segundo capítulo se describe el proceso metodológico. En el tercer apartado se muestran el análisis realizado a cada uno de los grupos que conforman los activos de las pequeñas empresas de la muestra frente a los requisitos de reconocimiento que contempla la NIIF para Pymes. Y finalmente, en el capítulo cuarto se describen las conclusiones del estudio.

\subsection{Revisión de la literatura}

Actualmente las empresas catalogadas en el grupo 2 que aplican la NIIF para Pymes, están en la etapa de transición, en el caso de las pequeñas empresas los estudios sobre los impactos que éstas pueden generar son escasas. Por ende se seleccionaron los siguientes estudios.

Como estudio de caso, en una mediana empresa de Bogotá las principales diferencias que causaron efectos en los estados financieros por la aplicación de NIIF para Pymes fueron: Cálculo y valor del impuesto diferido; Deterioro del valor de las inversiones; Cuentas por cobrar sin intereses y Propiedades, planta y equipo [13].

Igualmente, el estudio realizado sobre los efectos financieros en una cooperativa colombiana por la implementación de la NIIF para las Pymes en su balance de apertura, arrojó una disminución en el patrimonio. A consecuencia de la aplicación de los estándares relativos a los instrumentos financieros, debido a que gran parte de su capital social no cumple la definición de patrimonio que allí se establece al no estar considerado en su capital mínimo irreductible, por ende los aportes quedarán clasificados según lo indica la norma. Así mismo evidencian un efecto en la propiedad, planta y equipo como producto del uso del método de costo atribuido y de la reclasificación del anticipo de propiedad, planta y equipo que aparecía bajo el concepto de cuentas por cobrar bajo PCGA [14]. 
Análisis de los potenciales efectos en el reconocimiento de los activos de las pequeñas empresas de CúcutaColombia por la implementación de la NIIF para Pymes
Dentro de los estudios realizados por los organismos competentes sobre la temática, se muestra el documento de orientación técnica del CTCP [15] sobre la aplicación de la NIIF para Pymes en un caso de estudio. En cual se evidencia que el grupo de activos con mayor ajuste es la propiedad planta y equipo, dado que se adoptó la exención contemplada en la párrafo 35.10 literal (d) revaluación como costo atribuido, se reconoció un activo por arrendamiento financiero y se ajustó la depreciación según los criterios económicos y financieros. Así mismo se muestran cambios en el reconocimiento de la plusvalía, y retiros en activos intangibles por concepto de costos de investigación y desarrollo.

El estudio realizado por la superintendencia de sociedades [16] en uno de sus objetivos describe el impacto patrimonial en la elaboración del balance de apertura aplicando la NIIF para Pymes, en una muestra de pequeñas y medianas empresas del sector real colombiano ${ }^{2}$. El estudio se realizó desde dos puntos de vista: aplicación del modelo del costo y aplicación del modelo del valor razonable (costo atribuido). Se evidenció que los impactos son negativos en el patrimonio ${ }^{3}$ desde los dos puntos de vista, sin embargo la aplicación de las políticas de costo atribuido originan menores impactos patrimoniales que el uso del modelo del costo, en aquellos macro sectores que tienen grandes valorizaciones en los componentes de sus estados financieros. Igualmente, desde el punto de vista sectorial, Rodríguez [17] afirma que los impactos a generarse con la convergencia de NIIF para Pymes son los sectores de la manufactura y la de medios de comunicación principalmente en la propiedad planta y equipo y los activos intangibles.

\footnotetext{
${ }^{2}$ Dentro del estudio, el departamento de Norte de Santander, tiene una participación en la muestra de 4 empresas de las cuales una es pequeña empresa y pertenece al macro sector de minas y canteras. ${ }^{3}$ Los cambios en el patrimonio, se reflejan principalmente por los ajustes en los grupos de deudores y otras cuentas por cobrar, inventarios, propiedad planta y equipo y activos intangible que conforman los activos
}

Por otra parte, como referencia a la aplicación de la NIIF plenas en el grupo de activos diferidos en una empresa del sector hidrocarburos y derivados del petróleo, se evidenció que los actuales activos diferidos podrían convertirse en gastos del período, viendo deterioradas sus utilidades por este concepto, o incluso llegando a revelar pérdidas por esta misma razón, lo cual impacta de manera significativa partidas como el total de activos y resultados financieros, así como los indicadores de endeudamiento y rentabilidad del ente económico. En consecuencia, el término diferidos desaparecería del lenguaje normativo local y solo se podría hacer referencia a estas transacciones como gastos o como intangibles dependiendo de los requerimientos dados por las NIIF [18]. De igual manera en la NIIF para Pymes no mencionan ni hace referencia a ellos más allá de los llamados "impuestos diferidos", dando lugar a que los diferidos no se registren como un subrrubro independiente.

En este sentido, el presente artículo aporta a la literatura, al describir los posibles efectos en cuanto a reconocimiento de los activos en una muestra de pequeñas empresas en la ciudad de Cúcuta, partiendo del análisis de los requisitos que contempla la NIIF para pymes, en cuanto a la comprensión del hecho económico o la transacción, definición y reconocimiento de los activos y finalmente la clasificación en las secciones que contempla la misma. Así mismo ser insumo en futuras investigaciones en pequeñas empresas sobre la aplicación de la NIIF para Pymes.

\section{Materiales y métodos}

El trabajo se desarrolló por medio de una metodología descriptiva, dada que la misma conduce a presentar los hechos tal como ocurren y que caracterizan la realidad percibida, en relación con los cambios de la nueva normatividad contable aceptada en Colombia. 
Se identificó una población de 98 pequeñas empresas(Tabla II), Inspeccionadas ovigiladas por la superintendencia de sociedades, y que

Tabla II. Pequeñas empresas de la población.

\begin{tabular}{|c|c|}
\hline SECTOR ECONÓMICO & PEQUEÑA EMPRESA \\
\hline A. Explotación de Minas y Canteras & 12 \\
\hline B. Industria manufacturera & 15 \\
\hline C. Construcción & 33 \\
\hline D. Comercio al por mayor al por Menor & 1 \\
\hline E. Hoteles y restaurantes & 2 \\
\hline F. Trasportes, almacenamiento y Comunicaciones & 14 \\
\hline G. Actividades Inmobiliarias y Empresariales & 2 \\
\hline H. Educación & 3 \\
\hline I. Otras actividades de servicios comunitarios & 98 \\
\hline Total & \\
\hline
\end{tabular}

Fuente: Elaboración propia, a partir de la base de datos superintendencia de sociedades, intendencia regional Cúcuta.

Con el fin de conocer la percepción que tenían las pequeñas empresas de Cúcuta sobre el proceso de convergencia a las NIIF para Pymes, los autores Y. K. Criado, A.C. Rangel, E. Solano, después de aplicar una encuesta y analizar e interpretar la información respectiva de su estudio, obtuvieron los siguientes hallazgos "Los resultados reflejan el conocimiento que tienen los encargados del área contable y financiera sobre las NIIF para PYMES y la falta de claridad que tienen sobre algunos aspectos relacionados con los estados financieros que definen la etapa de transición y cuál es el procedimiento a seguir para converger de principios de contabilidad generalmente aceptados a norma internacional de información financiera para PYMES", [19], e igualmente, los autores. [20] y [21] en su investigación aplicaron un instrumento de recolección de datos (encuesta), que además permitiera identificar cuáles pequeñas empresas autorizaban conocer los estados financieros y demás documentos necesarios para realizar el análisis, finalmente estos trabajos sirvieron de insumo y la muestra que proporcionaron fue de 9 pequeñas empresas (Tabla III). Por ende, La información suministrada por las mismas, al 31 de diciembre de 2012, fue objeto de simulación para realizar una descripción cualitativa de cumplan con los requisitos contemplados en la tabla I, para pertenecer al grupo el 2 que aplican NIIF para Pymes. los hallazgos en términos de los efectos de la implementación de la NIIF para PYMES [22] en relación con los requisitos para el reconocimiento de los activos.

Tabla III. Empresas de la muestra.

\begin{tabular}{|c|c|}
\hline SECTOR ECONÓMICO & EMPRESAS \\
\hline Actividades inmobiliarias & 2 \\
\hline Actividades empresariales & 2 \\
\hline Explotación de minas y canteras & 1 \\
\hline Construcción & 1 \\
\hline Comercio al por mayor y al por menor. & 1 \\
\hline Manufacturera & 2 \\
\hline Total & 9 \\
\hline
\end{tabular}

La metodología para identificar los elementos del activo bajo NIIF para pymes en las pequeñas empresas fue la siguiente:

- Comprensión del hecho económico.

Julio - Dic. 2017

ISSN 0122-820X

-ISSN 2422-5053

PP: 116-130

- Definición de activo según el marco conceptual de la NIIF para Pymes párrafo 2.15 literal (a). La situación financiera de una empresa está relacionada con los activos, los pasivos, y el patrimonio. En el caso de los activos se definen: como un recurso controlado por la entidad como resultado de sucesos pasados, del que la entidad espera obtener, en el futuro, beneficios económicos. 
- Reconocimiento en el estado de situación financiera. El termino reconocimiento utilizado por la NIIF para Pymes en el párrafo 2.27 es referido al proceso de incorporación en los estados financieros de una partida que cumple con la definición de un activo, pasivo, ingreso, o gasto. El reconocimiento de un activo en el estado de situación financiera según párrafo 2.37 se determina: (i) cuando sea probable que del mismo se obtengan beneficios económicos futuros para la entidad, más allá del periodo actual sobre el que se informa, si ello no se cumple, esta transacción dará lugar al reconocimiento de un gasto en el estado de resultados integral, (i) y además, el activo tenga un costo o valor que pueda ser medido con fiabilidad

3. Clasificación del activo. Después de cumplir con los requisitos de definición y reconocimiento de activo, se identifica cual es la sección que establece los principios para clasificar cada elemento.

4. En el caso de una situación para la cual las secciones de la NIIF para Pymes no traten ese hecho económico, pero si cumple con la definición y reconocimiento de activo en el marco conceptual, se debe desarrollar una política contable para reconocerlos como otro activo.

\section{Resultados y discusión}

Siguiendo la metodología planteada anteriormente, en los activos de las pequeñas empresas $^{4}$ bajo Principios de Contabilidad Generalmente Aceptados PCGA a NIIF para Pymes, los resultados en cuanto a reconocimiento se reflejaron así:

- Disponible: Se consideró efectivo el dinero en caja, el cual a la fecha de transición no debería tener ningún ajuste, y los rubros en

${ }^{4} \mathrm{El}$ análisis se realizó partiendo de los grupos de activos que conforman las pequeñas empresas, sin generalizar en los sectores económicos a los cuales pertenecen. bancos, teniendo en cuenta las conciliaciones en notas, consignaciones, cheques., en especial este último por el manejo de los cheques girados y no cobrados, por ende es importante para la empresa fijar una política contable para el tratamiento de éstos.

- Inversiones: Las nversiones de corto plazo y de bajo riesgo con mayor grado de liquidez que se efectuaron por excedentes de efectivo $\mathrm{y}$ que posteriormente se fueran a utilizar para actividades operacionales de la empresa se reconocieron como equivalentes de efectivo, entre ellos el certificado a depósito a término CDT que mantenían tres pequeñas empresas, quedando dentro del alcance del párrafo 11.5 literal (b) de la NIIF para Pymes.

Se reconoció la compra en efectivo de las acciones ordinarias de otra entidad, dos pequeñas empresas tenían representadas acciones en sus activos, las cuáles presentaban evidencia objetiva de deterioro por dificultades financieras significativas del emisor o del obligado, según lo indica la norma se debe reconocer una pérdida por deterioro medida según el párrafo 11.25 literal (b) de la NIIF para Pymes. Estas empresas no lo estaban haciendo y los saldos no reflejaban la realidad económica.

- Cuentas por cobrar: Se reconocieron en las pequeñas empresas las cuentas por cobrar según el alcance de la sección 11 instrumentos financieros ${ }^{5}$ :

- Los clientes. Teniendo en cuenta el reconocimiento del deterioro del valor a recuperar bajo NIIF para Pymes mediante

\footnotetext{
${ }^{5}$ Según el párrafo 11.2 "un entidad optara entre aplicar: a) lo previsto en la sección 11 y sección 12 en su totalidad, o b) las disposiciones sobre reconocimiento y medición de la NIC 39 instrumentos financieros: reconocimiento $\mathrm{y}$ medición $\mathrm{y}$ los requerimientos de información a revelar de las secciones 11 y 12" seguidamente clarifica "para contabilizar todos sus instrumentos financieros. La elección de (a) o (b) por parte de un entidad es una elección de política contable." Para efectos del reconocimiento de los activos de las pequeñas empresas de la muestra se optó por utilizar el alcance y definición de la sección 11 .
} 
una evaluación financiera y comercial de cada uno de ellos.

- Los anticipos y avances. Según los ejemplos del párrafo 11.5 los anticipos y avances no no son instrumentos financieros, y al mismo tiempo no cumplen con las condiciones del párrafo 11.8 en los literales (a) a (d) por tanto no están dentro del alcance de la Sección 11. Dado este panorama, los anticipos y avances se pueden clasificar en una categoría de activos según la destinación, siempre y cuando la transacción sea altamente probable [23]. De igual manera, no hay una sección específica que trate los anticipos y avances, por tanto se reconoce como otro activo en el estado de situación financiera ya que cumple con la definición de activo en el marco conceptual. Mediante esta reflexión se reclasificaron así los anticipos y avances a proveedores y contratista que presentaban dos pequeñas empresas.

- Los préstamos a socios o accionistas, particulares y empleados. Se reconocieron como activos financieros dado que cumplen la condición del párrafo 11.5 literal (d) «préstamos por cobrar» según el párrafo 11.8 literal (b) son instrumentos de deuda que deben satisfacer las condiciones contempladas en el párrafo 11.9. Tres pequeñas empresas tenían en las cuentas por cobrar estos préstamos de los cuales se evidenció que en el estado de resultados no se estaban reconociendo los intereses, es importante mencionar que bajo PCGA no se da importancia a estos préstamos, y generalmente no se reflejan directamente en el estado de resultados, la NIIF para Pymes en el párrafo 11.13 describe " "Si el acuerdo constituye una transacción de financiación, la entidad medirá el activo financiero al valor presente de los pagos futuros descontados a una tasa de interés de mercado para un instrumento de deuda similar." En los términos anteriores, se recomendó a las pequeñas empresas hacer una evaluación sobre la recuperabilidad de los futuros flujos de efectivo y contabilizar estos préstamos como lo indica la norma. Esta característica se evidenció en el estudio de Salazar [13] quien identificó las cuentas por cobrar sin intereses, como una diferencia que causó efectos en los estados financieros al aplicar NIIF para Pymes.

- Cuentas de carácter fiscal: Por características de los PCGA las pequeñas empresas tenían rubros de carácter fiscal dentro del activo, representados en el grupo de deudores, mediante las cuentas de: Anticipo de renta y complementarios, anticipo de industria y comercio y Retención en la fuente. La NIIF para Pymes no contempla expresamente como reconocer y medir un impuesto, sin embargo estas son obligaciones que se generan en cumplimiento de la legislación tributaria local, por tanto estas partidas cumplen con la definición de activo y deben reconocerse como tal cuando se presenta la operación que les da origen en el ámbito comercial [24]. Por otro parte las pequeñas empresas deben calcular y reconocer el impuesto diferido por las diferencias temporarias entre la base fiscal y la NIIF para Pymes como se indica en el párrafo 29.3 literal (e).

- Inventarios: Las pequeñas empresas que por su objeto social mantienen rubros en inventarios, se reconocieron según párrafo 13.1 de la NIIF para Pymes "los mantenidos para la venta en el curso normal de las operaciones; en proceso de producción con vista a esa venta o en forma de materiales $y$ suministros, para ser consumidos en el proceso de producción, o en la prestación de servicios". Así mismo se debe considerar la verificación de su estado, la recuperabilidad, y el análisis de la composición de sus costos para excluir intereses, costos de producción no capitalizables, diferencia en cambio."

- Propiedad planta y equipo-PPyE: De acuerdo a cada una de las actividades de las pequeñas empresas, varían las partidas 
Análisis de los potenciales efectos en el reconocimiento de los activos de las pequeñas empresas de CúcutaColombia por la implementación de la NIIF para Pymes

representadas en PPyE. Se reconocieron como activos tangibles según la NIIF para Pymes párrafo 17.2 y 17.4 "los que se mantienen para el uso en la producción o suministro de bienes o servicios, para arrendarlos a terceros o con propósitos administrativos, y se esperan usar durante más de un periodo" y que sea "probable que la entidad obtenga los beneficios económicos futuros asociados con el elemento, y el costo del elemento puede medirse confiabilidad" entre ellos: terrenos, construcciones y edificaciones que no generan renta, equipo de oficina, equipo de computación y comunicación, considerando la necesidad de verificar su estado y recuperabilidad por uso o venta.

En el caso específico de la muestra, tres pequeñas empresas, tenían dentro de su activo valorizaciones por concepto de PPyE (terrenos, construcciones y edificaciones). Según los PCGA las valorizaciones se presentan por separado del costo, revelando en notas su composición. Así mismo se evidenció que cuatro pequeñas empresas tenían saldos de ajustes por inflación en PPyE, los cuales fueron eliminados en el 2006, por lo tanto no deberían estar reflejados en el balance general. Un tema importante en la fecha de transición es la medición de la PPyE y las exenciones que se pueden utilizar voluntariamente. Para esta situación, el párrafo 35.10 permite que la entidad utilice las exenciones del literal (c) $)^{6}$ y (d) ${ }^{7}$ al preparar sus primeros estados financieros conforme a la NIIF para Pymes. En resumen, las empresas pueden elegir tres opciones voluntarias en el momento de medir sus activos fijos en la fecha de transición. Primera opción, usar el costo de acuerdo a los requerimientos de la sección 17

\footnotetext{
${ }^{6}$ Valor razonable como costo atribuido. Una entidad que adopta por primera vez la NIIF puede optar por medir una PPyE en la fecha de transición a esta NIIF por su valor razonable, y utilizar este valor razonable como el costo atribuido en esa fecha.

${ }^{7}$ Revaluación como costo atribuido. Una entidad que adopta por primera vez la NIIF puede optar por utilizar una revaluación según los PCGA anteriores de una partida de PPyE en la Fecha transición a esta NIIF o en una fecha anterior, como el costo atribuido en la fecha de revaluación.
}

Propiedad, Planta y Equipo. Segunda, bajo los PCGA tomar cualquier ajuste razonable atribuido al bien, pueden ser los ajustes por inflación o valorizaciones. Y tercera, optar por el valor razonable. Cualquier exención es permitida siempre que corresponde a la realidad comercial y/o funcional de los bienes y es decisión de la pequeña empresa estimar los efectos que implica cualquier decisión.

En cuanto a la depreciación se evidenció que se registraba como una actividad sin mayor análisis, en tanto que los conceptos de importe depreciable, valor residual o vida útil, son pocos utilizados, sobre todo en la vida útil que se trabaja generalmente con el esquema tributario. Por el contrario la NIIF para Pymes hace énfasis en la realización de análisis financieros de los bienes.

Estos resultados son consistentes con los autores [13], [15], [16] y [17] quienes evidenciaron que la propiedad planta y equipo es uno de los grupos de los activos que más genera cambios en los estados financieros en la aplicación de la NIIF para Pymes, por la utilización de la exención del costo atribuido o el costo histórico.

- Intangibles y diferidos: se reconocieron los intangibles que cumplían con la definición de activo intangible y con los criterios de reconocimiento descritos en el párrafo 18.4 de la NIIF para Pymes. Por el contario los activos diferidos no se reconocieron por no cumplir con la definición de activo contemplada en el marco conceptual de la NIIF para Pymes, como lo hacen los PCGA, que reconocen los diferidos representados en las siguientes cuentas: gastos pagados por anticipado, cargos diferidos, costos de exploración por amortizar, costos de explotación y desarrollo, cargos por corrección monetaria diferida y amortización acumulada, lo anterior significa que las pequeñas empresas con rubros representados bajo este grupo deben reclasificarlos a intangible si cumplen con los requerimientos de la norma o eliminarlos. 
En las pequeñas empresas se observaron los siguientes casos:

- Tres pequeñas empresas tenían representados en el grupo de diferidos: gastos pagados por anticipado por concepto de seguros y fianzas, los cuales se consideró reclasificarlos a activos intangibles, bajo estos criterios, la NIIF para Pymes en el párrafo 18.16 dice que "no impide reconocer los anticipos como activos, cuando el pago por los bienes o servicios se haya realizado con anterioridad a la entrega de los bienes o prestación de los servicios". En el mismo orden de ideas, el ejemplo 4 del módulo 18 de la NIIF para Pymes describe: Una entidad que adquiere un seguro ha pagado para transferir el riesgo a un tercero y ese pago se debe reconocer como un activo (pago anticipado por servicios) en la fecha en que se efectúa y luego como un gasto en los resultados durante el periodo de cobertura del seguro" adicional a estos planteamientos de la norma, los autores [25] y [26] afirma que los gastos pagados por anticipado por cualquier concepto, se reconoce como activo, y que este se deriva del derecho a recibir bienes o servicios que tienen valor y no el bien o servicio respectivo.

- El software contable se encontró registrado en los activos diferidos e intangibles, se concluyó eliminar de los activos estos rubros ya que tiene una proporción insignificante dentro del activo, y su vida útil no corresponde a la realidad económica, ya que está determinada bajo el enfoque fiscal y no financiero. Adicionalmente se consideró llevar las actualizaciones como un gasto dentro del periodo.

- Una pequeña empresa tenía representado en la cuenta de cargos diferidos, elementos de ropería y lencería, loza y cristalería, cubiertería y platería, utensilios de cocina, etc., sobre estos elementos se optó por la decisión de eliminarlos o reclasificarlos a PPyE si cumplen con los criterios de definición y reconocimiento de la norma. Para el caso específico se podrían reclasificar, pero ante una evaluación técnica financiera para verificar su estado y su recuperabilidad por uso, posiblemente se tengan que eliminar, además su amortización se estaba registrando sobre los criterios fiscales y no financieros.

- Una pequeña empresa tenía en la partida de intangibles un rubro representado en bienes recibidos en leasing por concepto de una maquinaria, que se iban amortizando en cada periodo. Según la NIIF para Pymes párrafo 17.2 y 17.4 ese activo cumple con los criterios de definición y reconocimiento de un elemento de PPyE. Por lo tanto, se reclasificó y se reconoció un pasivo financiero por el concepto de leasing. Tal ajuste es consistente con el caso de estudio propuesto por el CTCP [15] que reconocieron el activo y el pasivo por concepto de arrendamiento financiero. Además es importante mencionar que el artículo 127-1 del estatuto tributario que permitía manejar el leasing financiero como leasing operativo tuvo vigencia en Colombia hasta el 2012, por lo tanto, incluso tributariamente hay que manejarlo como un activo.

- Una empresa dedicada a la explotación de carbón, presentó una característica particular, los cargos diferidos representaban el $62 \%$ del total del activo, por ende se optó por revisar la sección 34 actividades especiales, la cual describe en el párrafo 34.11 "Una entidad que utilice esta NIIF y se dedique a la exploración, evaluación o extracción de recursos minerales (actividades de extracción) contabilizará el desembolso por la adquisición o el desarrollo de activos tangibles o intangibles para su uso en actividades de extracción, aplicando la Sección 17 Propiedades, Planta y Equipo y la Sección 18 Activos Intangibles Distintos de la Plusvalía, respectivamente. Cuando una entidad tenga la obligación de desmantelar o trasladar un elemento o restaurar un emplazamiento, estas obligaciones y costos 
Análisis de los potenciales efectos en el reconocimiento de los activos de las pequeñas empresas de CúcutaColombia por la implementación de la NIIF para Pymes se contabilizarán según la Sección 17 y la Sección 21 Provisiones y Contingencias". Igualmente el concepto $\mathrm{N}^{\mathrm{o}} 068$ emitido por el CTCP [27] ratifica que se pueden usar la sección 17 y 18 solo si cumple con los requisitos de definición y reconocimiento de las mismas.

Frente a lo expuesto, se determinó reconocer el rubro de estudios, investigaciones y proyectos como un intangible el cual está representado en un 56\% del total del activo, y tomando la exención de la NIIF para Pymes párrafo 35.10 , literal $\mathrm{b}$, que permite sobre las actividades de extracción lo siguiente: Una entidad que adopta por primera vez la NIIF, y utiliza la contabilidad de costo completo conforme a PCGA anteriores, puede optar por medir los activos de petróleo y gas (activos empleados en la exploración, evaluación, desarrollo o producción de petróleo y gas) en la fecha de transición a la NIIF para las PYMES, por el importe determinado según sus PCGA anteriores. La entidad comprobará el deterioro del valor de esos activos en la fecha de transición a esta NIIF, de acuerdo con la Sección 27 Deterioro del Valor de los Activos. Seguidamente, los gastos de organización y preoperativos representados en el 6\% del activo se eliminaron. Este resultado es consistente con el estudio de Torres y Sánchez [18] quienes en la aplicación de las NIIF plenas en una empresas del sector de hidrocarburos y derivados del petróleo evidenciaron que los activos diferidos podrían convertirse en gastos del período o ser reclasificados en intangibles, según el análisis de cada una de las partidas que integran este grupo de activos. Esta situación permite concluir que la aplicación de NIIF plenas y NIIF para pymes para estos conceptos es el mismo.

- Valorizaciones: Cuatro pequeñas empresas tenían valorizaciones en PPyE por concepto de terrenos, teniendo en cuenta que este rubro no cumple con la definición de activo, se optó por utilizar en la fecha de transición a la NIIF para Pymes la exención del párrafo 35.10 literal (d) "Revaluación como costo atribuido" y de esta manera reclasificar a cada rubro correspondiente en el activo. Sin embargo es una decisión de la pequeña empresa estimar los efectos en el reconocimiento y medición de la PPyE, frente a las exenciones que tiene la posibilidad de elegir voluntariamente en la fecha de elaboración del ESFA.

\section{Conclusiones}

En este estudio se evidenciaron hallazgos de la aplicación por primera vez de la NIIF para Pymes en el reconocimiento de los activos. Los hechos se reflejan en una muestra específica de 9 pequeñas empresas con diferentes actividades económicas en su objeto social, con el fin de tener un panorama con diversos escenarios. Sin embargo estos resultados no son generalizables ya que cada empresa tiene situaciones particulares.

Los efectos en cuanto a reconocimiento de los activos de las pequeñas empresas se reflejaron principalmente en:

- Propiedad planta y equipo, dado que las empresas que apliquen NIIF para Pymes pueden optar por utilizar voluntariamente las exenciones de costo atribuido, a diferencia de la NIIF plenas que exige medir la PPyE al valor razonable. Esto le permite a las pequeñas empresas realizar un análisis y revisar desde el punto de vista fiscal, como resolver los efectos que tendría en el futuro dado que en la fecha de transición no es obligatorio utilizar el valor razonable.

- Depuración de los diferidos. Dado que muchos de los conceptos de este grupo no cumplen con la definición de activo, si no se eliminan deben reclasificarse a intangibles siempre que cumplan con las condiciones de reconocimiento 
- Valorizaciones. por su efecto de reclasificar o eliminar, según la política contable que elija la pequeña empresa.

Frente a estos posibles efectos las pequeñas empresas deben analizar las decisiones al momento de elaborar las políticas contables, dado que una disminución en los activos, puede afectar incluso su financiamiento ${ }^{8}$, desde otro aspecto a considerar esta la parte impositiva ya que "podría afectar de manera importante las bases fiscales o generar importantes diferencias entre el importe en libros de un activo y sus bases fiscales" [16]. En este sentido, las pequeñas empresas deben depurar las cifras representadas en sus activos, considerando su definición y reconocimiento mediante un análisis de su estado, su recuperabilidad, si genera beneficios, efectos futuros y siempre prevaleciendo la realidad del hecho económico. Esto implica un equipo de trabajo que involucra no solo área contable y financiera, sino a la organización en general.

Por otra parte, la contabilidad colombiana debe cambiar el modelo orientado a cumplir con las obligaciones tributarias y pasar a un modelo que ejercita la capacidad de juicios y estimaciones para reconocer una transacción o suceso económico que mejore la calidad de la información contable y financiera. Los principios y normas de contabilidad aplicados en Colombia no son ajenos a la contabilidad internacional solo se dejaron de actualizar, pero en ello no radican esencialmente los cambios, la principal dificultad es que los hechos económicos estaban reconocidos $\mathrm{y}$ registrados según los requerimientos de la legislación tributaria, que no corresponde a la realidad económica de la empresa, por ende, la NIIF para Pymes reconoce el impuesto diferido que surge de diferencias temporarias entre las bases fiscales y tributarias.

${ }^{8}$ Por los cambios en los indicadores financieros (Endeudamiento, capital de trabajo) que pueda generar la aplicación de la NIIF para Pymes.
Finalmente este estudio muestra las implicaciones que generarían estos cambios en una futura fecha de transición obligatoria a la NIIF para Pymes, pero al mismo tiempo evidencia el mejoramiento de la calidad de la información contable y financiera.

\section{Agradecimientos}

A la universidad Francisco de Paula Santander, a los Empresarios, Contadores y Asesores Financieros de la PYMES que participaron en esta investigación y al Fondo de Investigaciones Universitarias FINU.

\section{Referencias}

[1] Congreso de la República, Decreto 2649, "por la cual se reglamenta la contabilidad en general y se expide los principios o normas de contabilidad generalmente aceptadas en Colombia, Bogotá, D.C., 29 diciembre. 1993. [En línea] Disponible en: http://www. alcaldiabogota.gov.co/sisjur/normas/ Norma1.jsp?i=9863 [Accedido: 1-jul2016]

[2] Congreso de la República, decreto 2650, "por el cual se modifica el plan único para comerciantes," Bogotá, D.C., 29 diciembre. 1993. [En línea] Disponible en: http://incp.org.co/ Site/2012/ legislativa/2650.pdf [Accedido: 15ago-2016]

[3] Congreso de la República, ley 550, "por la cual se establece un régimen que promueva y facilite la reactivación empresarial y la reestructuración de los entes territoriales para asegurar la función social de las empresas y lograr el desarrollo armónico de las regiones y se dictan disposiciones para armonizar el régimen legal vigente con las normas de esta ley.” Bogotá, D.C. 30 diciembre. 1999. [en línea] Disponible en: ww.minhacienda.gov.co/ portal/
Julio - Dic. 2017

ISSN 0122-820X

E-ISSN 2422-5053

PP: 116-130 
page/portal/HomeMinhacienda/ presupuestogeneraldelanacion/ Normativapresupuesto/Leyes/Ley 550 de diciembre 30 de 1999 [Accedido: 18-ago-2016]

[4] Comité Interinstitucional, "Proyecto de ley de intervención económica por medio de la cual se señalan los mecanismos por los cuales se adoptan en Colombia los estándares internacionales de contabilidad, auditoría y contaduría, se modifican el Código de Comercio, la normatividad contable y se dictan otras disposiciones relacionadas con la materia." Bogotá, D.C.,10 diciembre. 2003. [En línea] Disponible en: http://www.javeriana. edu.co/ personales/hbermude/ leycontable/ contadores/2003anteproyectodeleyIntervencion.pdf [Accedido: 28-ago-2016]

[5] Congreso de la República, Proyecto de ley 165,"Por la cualelEstadocolombiano adopta las Normas Internacionales de Información Financiera para la presentación de informes contables." Bogotá, D.C., 30 diciembre. 2007. [En línea] Disponible en: http://www. javeriana.edu.co/personales/hbermude/ leycontable/contadores/2008ponencia $165 \mathrm{C} \% 282 \% 29 . p d f$ [Accedido: 25-ago-2016]

[6] Congreso de la República, ley 1314, "por la cual se regulan los principios y normas de contabilidad e información financiera $y$ de aseguramiento de información aceptados en Colombia, se señalan las autoridades competentes, el procedimiento para su expedición y se determinan las entidades responsables de vigilar su cumplimiento," Bogotá, D.C., 13 julio. 2009. [En línea] Disponible en: http://www.secretariasenado.gov. co/ senado/basedoc/ley_1314_2009. html [Accedido: 30-ago-2016]
[7] Consejo Técnico de la Contaduría Pública, "Direccionamiento estratégico del proceso de convergencia de las normas de contabilidad e información financiera $y$ de aseguramiento de la información, con estándares internacionales" 2012, Bogotá, D.C. [En línea] Disponible en: http://www. ctcp. gov.co/documentos2.php [Accedido: 12-sep-2016]

[8] Ministerio de Comercio, Industria y Turismo, Decreto 3022, "Por el cual se reglamenta la Ley 1314 de 2009 sobre el marco técnico normativo para los preparadores de información financiera que conforman el Grupo 2,'Bogotá. D.C., 27, Diciembre. 2013. [En línea] Disponible en: www.mincit. gov.co/ descargar.php? $\mathrm{id}=69284 \quad$ [Accedido: 10-sep-2016]

[9] Actualisece.com "Cronograma para aplicación de Estándares NIIF. [En línea] Disponible en: http://actualicese. com/ modelos-y-formatos/guia-cronogramapara-aplicacion-de-estandaresniif/ [Accedido: 27-ago-2016]

[10] Superintendencia de sociedades, circular 115-000002, "proceso de convergencia a Normas de Información Financiera para los preparadores que conforman el grupo 2,"Bogotá. D.C., 05, Marzo. 2014. [En línea] Disponible en: http://www.supersociedades.gov. co/asuntos-economicos-y-contables/ procesos-de-convergencia-niifs/ circulares/Documents/Circular\%20 Externa\%20115-000002\%20de\%20 2014.pdf [Accedido: 10-sep-2016]

[11] Ministerio de Comercio, Industria y Turismo, Decreto 2267, "Por el cual se modifican parcialmente los decretos 1851 y 3022 de 2013 y se dictan otras disposiciones.'Bogotá. D.C., 11,Noviembre. 2014. [En línea] Disponible en: http:// 
www.minhacienda.gov.co/portal/ page/portal/HomeMinhacienda/ elministerio/NormativaMinhacienda/ Decretos/2014/DECRETO\%20 $2267 \% 20$ DEL $\% 2011 \% 20$ DE $\% 20$ NOVIEMBRE\%20DE\%202014.pdf [Accedido: 12-sep-2016]

[12] Ministerio de Comercio, Industria y Turismo, Decreto 2420, "Compila los decretos reglamentarios de la Ley 1314 del 2009 con respecto a NIIF y NAI: Decretos 2706 del 2012, 2784 del 2012, 3022 del 2013 y 302 del 2015" Bogotá. D.C., 11, Noviembre. 2014. [En línea] Disponible en: http://wp.presidencia. gov.co/sitios/normativa/decretos/2015/ Decretos $2015 /$ DECRETO \% 20 $2420 \% 20$ DEL $\% 2014 \% 20$ DE $\% 20$ DICIEMBRE\%20DE\%202015\%20 -\%20copia.pdf [Accedido: 1-oct-2016]

[13] É.E. Salazar, "Efectos de la implementación de la NIIF para las PYMES en una mediana empresa ubicada en la ciudad de Bogotá," Cuadernos de Contabilidad, vol. 14, no 35, pp. 395-414, 2013.

[14] C.E.Castaño, J.E.Zamarray J.A.Correa, "Efectos financieros en una cooperativa colombiana por la implementación de estándares internacionales de información financiera para las pyme en su balance de apertura", Cuadernos de Contabilidad, vol. 15, no 38, pp. 427458, 2014.

[15] Consejo Técnico de la Contaduría Pública, documento de orientación técnica 005 "adopción por primera vez de las NIIF para Pymes" 2015, Bogotá, D.C.

[16] Superintendencia de sociedades "Diagnóstico sobre los impactos contables y financieros de los estándares internacionales de contabilidad e información financiera en las empresas del sector real".

Bogotá. [En línea]. Disponible: http://www.supersociedades.gov. co/asuntoseconomicos-y-contables/ procesosde-convergencia-niifs/ estadisticade-la-convergencia/ Documents/Anexo_NIF_2_Marzo Documento Base Fase IV\% $\overline{5} \mathrm{~B} 1 \% 5 \mathrm{D}$. pdf [Accedido: 10-oct-2016]

[17] D.C. Rodríguez, "Cómo afectará la aplicación de las normas internacionales de contabilidad a las pymes en Colombia", Apuntes Contables, no. 17, pp. 59-110, 2014.

[18] M.A. Torres y M. Sánchez, "Impacto en la presentación de los estados financieros por el tratamiento contable de los diferidos bajo normas internacionales de contabilidad en Colombia," Gestión y Sociedad, vol. 5 no 2, pp. 147-179, 2012.

[19] Y.K. Criado, A.C. Rangel y E. Solano, "Estudio de las pequeñas empresas de Cúcuta sobre la convergencia a las normas internacionales de información financiera para pymes", Respuestas, vol. 19, no.2, pp. 6. 2014.

[20] J. Álvarez, "Percepción de las pequeñas empresas de explotación de minas y canteras, de construcción e industria manufacturera de Cúcuta sobre la convergencia a las normas internacionales de información financiera (NIIF) para las pymes," trabajo de fin de grado, Universidad Francisco de Paula Santander, Cúcuta 2013. Vol. 22 2

Julio - Dic. 2017 ISSN 0122-820X -ISSN 2422-5053 PP: 116-130

\section{.}

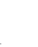


[22] Norma Internacional de Información Financiera (NIIF) para Pequeñas y Medianas Entidades (PYMES), Madrid, España: IFRS, 2009.

[23] J.D. Maya, "Anticipos y avances: tratamiento en NIIF para pymes" [En línea] actualícese.com. Disponible en: http://actualicese. com/ actualidad/2015/10/21/anticiposyavances-tratamiento-en-niifparapymes/ [Accedido: 19-sep-2016]

[24] J.D. Maya, "Anticipos de impuestos e impuestos por pagar: reconocimiento en NIIF” [En línea] actualícese.com. Disponible en: http://actualicese. com/ actualidad/2015/12/16/ anticipos-deimpuestos-e-impuestospor-pagarreconocimiento-en-niif/ [Accedido: 21-oct-2016]

[25] Superintendencia de sociedades, "guía práctica para elaborar el estado de situación financiera de apertura bajo NIIF para PYMES" [En línea]. Disponible en: http://www. supersociedades.gov.co/asuntoseconomicos-y-contables/documents/ GuiaPractica.pdf [Accedido: 6-nov2016]

[26] É.E. Salazar, "Gastos pagados por anticipado" Contrapartida, no. 1364, 2015.

130

grado, Universidad Francisco de Paula Santander, Cúcuta, 2013. 